\title{
Efeitos do carbono na evolução de segundas-fases e na densificação do nitreto de alumínio com $\mathrm{Y}_{2} \mathrm{O}_{3}$
}

\section{(Effects of carbon on second-phase evolution and densification of aluminum nitride with $\mathrm{Y}_{2} \mathrm{O}_{3}$ )}

\author{
A. L. Molisani ${ }^{1}$, H. Goldenstein ${ }^{1}, H . N$. Yoshimura ${ }^{2}$ \\ ${ }^{1}$ Escola Politécnica da Universidade de S. Paulo, Av. Prof. Mello Moraes 2463, S. Paulo, SP 05508-900 \\ ${ }^{2}$ Universidade Federal do ABC, Rua Santa Adélia 166, Santo André, SP 09210-170 \\ humberto.yoshimura@ufabc.edu.br,amolisani@gmail.com
}

\begin{abstract}
Resumo
Foi investigado o efeito da adição do carbono na evolução de segundas-fases e na densificação do $\mathrm{AlN}$ com $4 \%$ em massa de $\mathrm{Y}_{2} \mathrm{O}_{3}$. A mudança de composição da segunda-fase do $\mathrm{AlN}$ com $\mathrm{Y}_{2} \mathrm{O}_{3}$ foi induzida pela adição de $0,5 \%$ e $1,0 \%$ em massa de carbono. A sinterização sob atmosfera de nitrogênio foi realizada em forno com elemento resistivo de tungstênio entre $1650{ }^{\circ} \mathrm{C}$ e $1850{ }^{\circ} \mathrm{C}$. A evolução de segunda-fase mostrou uma tendência para formar fases mais ricas em ítrio com o aumento do teor de carbono, $\mathrm{o}$ que atrasou a densificação do $\mathrm{AlN}$ com $\mathrm{Y}_{2} \mathrm{O}_{3}$ devido ao aumento da temperatura de formação de fase líquida. O efeito prejudicial causado pela adição de carbono diminuiu com o aumento da temperatura de sinterização, pois todas as amostras atingiram quase completa densificação após sinterização a $1800^{\circ} \mathrm{C}$. A adição de carbono induziu uma evaporação significativa de compostos durante a sinterização, mas o comportamento de densificação foi pouco influenciado pela redução na fração de fase líquida existente na temperatura de sinterização e pelo gás aprisionado no interior dos poros fechados.
\end{abstract}

Palavras-chave: $\mathrm{AlN}, \mathrm{Y}_{2} \mathrm{O}_{3}$, carbono, sinterização, segundas-fases.

Abstract

The effect of carbon addition on the second-phases evolution and on the densification of AlN with $4 w t . \% . Y_{2} \mathrm{O}_{3}$ was investigated. The change of second-phase composition of AlN with $\mathrm{Y}_{2} \mathrm{O}_{3}$ was induced by the addition of 0.5 wt.\% and 1.0 wt.\% carbon. Sintering under nitrogen atmosphere was performed in tungsten heated furnace between $1650{ }^{\circ} \mathrm{C}$ and $1850{ }^{\circ} \mathrm{C}$. The second-phase evolution had a tendency to form Y-richer phases with increasing carbon content, which delayed the densification of $\mathrm{AlN}$ with $\mathrm{Y}_{2} \mathrm{O}_{3}$ due to the increase of liquid-phase formation temperature. The harmful effect caused by the addition of carbon was diminished with increasing sintering temperature, since all samples achieved almost full densification after sintering at $1800^{\circ} \mathrm{C}$. The addition of carbon induced significant evaporation of compounds during sintering, but the densification behavior was little influenced by the reduction in liquid-phase fraction formed at sintering temperature or the entrapped gas inside the closed pores.

Keywords: $\mathrm{AlN}, \mathrm{Y}_{2} \mathrm{O}_{3}$, carbon, sintering, second-phases.

\section{INTRODUÇÃO}

A cerâmica de nitreto de alumínio (AIN) tem potenciais aplicações como substrato e material de encapsulamento para dispositivos eletrônicos, pois apresenta alta condutividade térmica, elevada resistência à ruptura dielétrica, baixas perdas em alta freqüência, elevada resistividade elétrica e coeficiente de expansão térmica $\left(4,4 \times 10^{-6} /{ }^{\circ} \mathrm{C}\right.$ entre $20{ }^{\circ} \mathrm{C}$ e $\left.200{ }^{\circ} \mathrm{C}\right)$ próximo daquele do silício $\left(3,2 \times 10^{-6} /{ }^{\circ} \mathrm{C}\right.$ entre $20{ }^{\circ} \mathrm{C}$ e $200{ }^{\circ} \mathrm{C}$ ), que é um dos principais materiais usados pela indústria de microeletrônica para a fabricação de chips $[1,2]$. Além disso, apresenta boas propriedades mecânicas, próximas das cerâmicas de alumina $[3,4]$. Assim, a cerâmica de AlN é uma excelente candidata para substituir as cerâmicas de alumina $\left(\mathrm{Al}_{2} \mathrm{O}_{3}\right)$ e berília $(\mathrm{BeO})$ na fabricação de dispositivos semicondutores. Adicionalmente, o AlN pode ser empregado na fabricação de absorvedor de calor (heat sink), espalhador de calor para laser, ferramenta de corte, componente para sistema óptico-eletrônico e cadinho de fundição [2].

A superfície das partículas do pó de AlN apresenta uma camada de óxido amorfo ou hidróxido de alumínio [5, 6], decorrente da reação de hidrólise causada pela umidade do meio ambiente. Durante a sinterização, o oxigênio desta camada entra em solução sólida na rede cristalina do AlN, o que leva à formação de vacâncias de alumínio devido à compensação de carga gerada pela substituição de nitrogênio por oxigênio [7]. A presença de vacâncias de alumínio melhora a densificação do AlN devido ao aumento de seu coeficiente de autodifusão [1,2]. Por outro lado, a condutividade térmica é diminuída drasticamente com o aumento da concentração de vacâncias de alumínio, pois os 
fônons, responsáveis pela condução de calor em materiais cerâmicos, têm seu livre caminho médio diminuído pela interação fônon-vacância $[2,8]$.

Inicialmente, os aditivos de sinterização foram usados para promover a densificação do AlN em menores temperaturas por sinterização via fase líquida [9], pois é difícil ocorrer densificação nesta cerâmica devido à sua natureza covalente. Adicionalmente, sua condutividade térmica melhorou significativamente com o uso de aditivos de sinterização, pois o aditivo reage com o oxigênio (na forma de $\mathrm{Al}_{2} \mathrm{O}_{3}$ ) da superfície das partículas de AlN, formando segundas-fases de aluminato [10]. Deste modo, o oxigênio é incorporado às segundas-fases de contorno de grão, o que evita ou minimiza sua solubilização na rede cristalina do AlN. Os óxidos de alcalinos terrosos e de terras raras são usados habitualmente como aditivos de sinterização, mas a ítria $\left(\mathrm{Y}_{2} \mathrm{O}_{3}\right)$ se tornou mais popular porque o $\mathrm{AlN}$ preparado com este aditivo pode apresentar elevados valores de condutividade térmica (180$230 \mathrm{~W} / \mathrm{mK})[2]$.

A cerâmica de AlN é freqüentemente sinterizada em forno com elemento resistivo de grafita e sob atmosfera de gás nitrogênio. Deste modo, estabelece-se uma atmosfera de nitrogênio parcialmente redutora durante a sinterização, que é considerada como um dos mecanismos responsáveis pela melhora da condutividade térmica do AlN [11-15]. A atmosfera redutora promove a desoxidação das fases de contorno de grão, o que melhora a condutividade térmica devido à "limpeza" (cleanness) da microestrutura por meio da diminuição da fração de segundas-fases de baixa condutividade térmica [11, 15-18]. Embora haja um consenso para utilizar uma atmosfera redutora na otimização da condutividade térmica do AlN com $\mathrm{Y}_{2} \mathrm{O}_{3}$, observou-se que a presença de uma atmosfera fortemente redutora pode inibir a densificação e, assim, diminuir drasticamente a condutividade térmica [18]. Há procedimentos comumente usados para impedir a formação de uma atmosfera excessivamente redutora, tais como [18]: utilização de cadinho de nitreto de boro (BN) ou cadinho de grafita com uma camada protetora de pó de nitreto de boro $(\mathrm{BN})$ ou de pó de AlN.

Algumas reações químicas, que descrevem o processo de desoxidação e nitretação das segundas-fases durante a sinterização sob atmosfera redutora de monóxido de carbono, foram propostas para o $\mathrm{AlN}$ com $\mathrm{Y}_{2} \mathrm{O}_{3}[11]$ :

$$
\begin{aligned}
& \mathrm{Al}_{2} \mathrm{O}_{3(\mathrm{~s})}+\mathrm{N}_{2(\mathrm{~g})}+3 \mathrm{CO}_{(\mathrm{g})} \leftrightarrow 2 \mathrm{AlN}_{(\mathrm{s})}+3 \mathrm{CO}_{2(\mathrm{~g})} \\
& \mathrm{Al}_{2} \mathrm{Y}_{4} \mathrm{O}_{9(\mathrm{~s})}+\mathrm{N}_{2(\mathrm{~g})}+3 \mathrm{CO}_{(\mathrm{g})} \leftrightarrow 2 \mathrm{AlN}_{(\mathrm{s})}+2 \mathrm{Y}_{2} \mathrm{O}_{3(\mathrm{~s})}+3 \mathrm{CO}_{2(\mathrm{~g})} \\
& \mathrm{Y}_{2} \mathrm{O}_{3(\mathrm{~s})}+\mathrm{N}_{2(\mathrm{~g})}+3 \mathrm{CO}_{(\mathrm{g})} \leftrightarrow 2 \mathrm{YN}_{(\mathrm{s})}+3 \mathrm{CO}_{2(\mathrm{~g})}
\end{aligned}
$$

Parte do gás $\mathrm{CO}_{2}$ produzido durante o processo de desoxidação das segundas-fases (reações $\mathrm{A}$ a $\mathrm{C}$ ) pode ser convertida em gás $\mathrm{CO}$ devido à presença de carbono proveniente da grafita do cadinho e do elemento de aquecimento do forno. Deste modo, a atmosfera redutora pode ser mantida durante a sinterização por meio da reação:

$$
\mathrm{CO}_{2(\mathrm{~g})}+\mathrm{C}_{(\mathrm{s})} \leftrightarrow 2 \mathrm{CO}_{(\mathrm{g})}
$$

Alguns trabalhos propuseram que, durante a sinterização sob atmosfera redutora, as fases de aluminato de ítrio presentes no interior do corpo consolidado podem ser decompostas em $\mathrm{Al}_{2} \mathrm{O}_{3}$ e $\mathrm{Y}_{2} \mathrm{O}_{3}$ e, então, ao migrarem para a superfície do corpo sofrem desoxidação e nitretação, conforme previsto pelas reações A e C [15].

Outra forma utilizada para aumentar a condutividade térmica do AlN com $\mathrm{Y}_{2} \mathrm{O}_{3}$ é o uso de pó de grafita ou de negro de fumo como aditivo de sinterização $[12,18$, 19]. O aumento da condutividade térmica é decorrente da diminuição da fração de segundas-fases, que ocorre devido ao processo de desoxidação por carbono. Yan et al. [20] propuseram que o carbono reage preferencialmente com o oxigênio (na forma de $\mathrm{Al}_{2} \mathrm{O}_{3}$ ) presente na superfície do pó de $\mathrm{AlN}$ (reação $\mathrm{E}$ ), pois o $\mathrm{Y}_{2} \mathrm{O}_{3}$ é mais estável termodinamicamente do que $\mathrm{o}_{2} \mathrm{Al}_{2} \mathrm{O}_{3}$. Outros trabalhos também indicaram que a adição de carbono induz a desoxidação da superfície do pó de AlN por meio da reação $[19,21]$ :

$\mathrm{Al}_{2} \mathrm{O}_{3(\mathrm{~s})}+3 \mathrm{C}_{(\mathrm{s})}+\mathrm{N}_{2(\mathrm{~g})} \leftrightarrow 2 \mathrm{AlN}_{(\mathrm{s})}+3 \mathrm{CO}_{(\mathrm{g})}$

Como o $\mathrm{Al}_{2} \mathrm{O}_{3}$ é essencial para formar a fase líquida e promover a densificação do AlN, Yan et al. [20] propuseram que há uma condição limite que possibilita a densificação do AlN com $\mathrm{Y}_{2} \mathrm{O}_{3}$ na presença de carbono, dada pela razão molar entre o carbono adicionado e o teor de oxigênio do pó de AlN. Segundo os autores [20], a densificação do AlN somente ocorre na condição em que a razão $\mathrm{C} / \mathrm{O}$ é menor do que 1 , já que na condição $\mathrm{C} / \mathrm{O}>1$ todo $\mathrm{o} \mathrm{Al}_{2} \mathrm{O}_{3}$ reage com o carbono (reação $\mathrm{E}$ ) e não há alumina remanescente para reagir com o $\mathrm{Y}_{2} \mathrm{O}_{3}$ para formar aluminatos de ítrio de baixo ponto de fusão. Neste caso, observa-se que o aumento do teor de carbono induz à diminuição da fração de $\mathrm{Al}_{2} \mathrm{O}_{3}$ presente no pó de AlN, o que resulta na formação de aluminatos mais ricos em $\mathrm{Y}_{2} \mathrm{O}_{3}$ ou na completa inibição da formação de aluminatos de ítrio.

Por outro lado, foi proposto que o processo de desoxidação e nitretação durante a sinterização do AlN com adição simultânea de $\mathrm{Y}_{2} \mathrm{O}_{3}$ e C pode ocorrer de acordo com as reações $\mathrm{E}$ a $\mathrm{G}$ [12]:

$$
\begin{aligned}
& \mathrm{Al}_{2} \mathrm{Y}_{4} \mathrm{O}_{9(\mathrm{~s})}+\mathrm{N}_{2(\mathrm{~g})}+3 \mathrm{C}_{(\mathrm{s})} \leftrightarrow 2 \mathrm{AlN}_{(\mathrm{s})}+2 \mathrm{Y}_{2} \mathrm{O}_{3(\mathrm{~s})}+3 \mathrm{CO}_{(\mathrm{g})} \\
& \mathrm{Y}_{2} \mathrm{O}_{3(\mathrm{~s})}+\mathrm{N}_{2(\mathrm{~g})}+3 \mathrm{C}_{(\mathrm{s})} \leftrightarrow 2 \mathrm{YN}_{(\mathrm{s})}+3 \mathrm{CO}_{(\mathrm{g})}
\end{aligned}
$$

Neste mecanismo considera-se que a formação dos aluminatos de ítrio ocorre antes da desoxidação e nitretação do $\mathrm{Al}_{2} \mathrm{O}_{3}$ (reação $\mathrm{E}$ ) ou ocorre simultaneamente com a reação $\mathrm{E}^{2}[12]$. Em seguida, os aluminatos de ítrio são desoxidados pelo carbono, conforme as reações $\mathrm{F}$ e G. Neste caso, a formação dos aluminatos de ítrio ocorre independente da adição ou não de carbono, o que difere do mecanismo proposto em [20]. Estes resultados mostram que o mecanismo de evolução das segundas-fases durante 
a sinterização do AlN com adição simultânea de $\mathrm{Y}_{2} \mathrm{O}_{3}$ e C não está completamente estabelecido. Assim, o objetivo deste trabalho é investigar o mecanismo de evolução das segundas-fases do AlN com $\mathrm{Y}_{2} \mathrm{O}_{3}$, onde a mudança de composição é induzida pela adição de pó de grafita. Além disso, é investigado o efeito da composição das segundasfases na densificação do AlN com $\mathrm{Y}_{2} \mathrm{O}_{3}$.

\section{EXPERIMENTAL}

As matérias-primas usadas na preparação dos corpos sinterizados foram: pó de nitreto de alumínio (AlN, grau F, Tokuyama Soda, Japão), pós de $\mathrm{Y}_{2} \mathrm{O}_{3}$ (Aldrich Chem. Co., Alemanha) e de grafita (C, grafite de lubrificação, Brasil) como aditivos de sinterização, polietileno glicol (PEG, Nacalai Tesque, Japão) como ligante, álcool isopropílico PA como meio de mistura, e suspensão de nitreto de boro (BN, Union Carbide, EUA), aplicado como revestimento do cadinho. Quatro composições de pós foram investigadas (\% em massa): 1) AlN sem aditivo, 2) AlN com 4\% $\mathrm{Y}_{2} \mathrm{O}_{3}$ (amostra 4Y), 3) AlN com $4 \% \mathrm{Y}_{2} \mathrm{O}_{3}$ e $0,5 \%$ de $\mathrm{C}$ (amostra 4Y05C), e 4) AlN com 4\% $\mathrm{Y}_{2} \mathrm{O}_{3}$ e $1,0 \%$ de $\mathrm{C}$ (amostra 4Y1C). Os pós de partida foram misturados em álcool isopropílico para evitar o problema de hidrólise do pó de AlN em ambiente aquoso. A dispersão de pós foi preparada em recipiente cilíndrico de material polimérico com 300 $\mathrm{mL}$ de álcool isopropílico, $2 \%$ em massa de PEG e $250 \mathrm{~g}$ de esferas de material polimérico (diâmetro $6 \mathrm{~mm}$ ) para $100 \mathrm{~g}$ de material sólido. A mistura da dispersão de pós foi realizada em um moinho de bolas (Yamato, UB32, Japão) com velocidade de $80 \mathrm{rpm}$ por $24 \mathrm{~h}$. A secagem foi realizada em uma placa quente a $100{ }^{\circ} \mathrm{C}$ por $12 \mathrm{~h}$ e a granulação foi realizada em peneira com tela de náilon de 80 mesh. A conformação foi realizada em duas etapas: prensagem uniaxial com pressão de $10 \mathrm{MPa}$ por $30 \mathrm{~s}$ (Gardelin, Brasil) seguida de prensagem isostática a frio com pressão de $150 \mathrm{MPa}$ por $15 \mathrm{~s}$ (Yuken Rubber Press, YRDW-26-100-R, Japão). O corpo compactado apresentou aproximadamente $15 \mathrm{~mm}$ de diâmetro e $5 \mathrm{~mm}$ de espessura. A sinterização foi realizada entre $1650{ }^{\circ} \mathrm{C}$ e $18500^{\circ} \mathrm{C}$ por 1 h sob fluxo de gás nitrogênio $(\sim 2 \mathrm{~L} / \mathrm{min})$ em forno com elemento resistivo de tungstênio (Nems, NM 15, Japão), com taxas de aquecimento e resfriamento de 10 e $30{ }^{\circ} \mathrm{C} /$ min, respectivamente. Um patamar intermediário a 500 ${ }^{\circ} \mathrm{C}$ por $0,5 \mathrm{~h}$ foi realizado para eliminação do ligante. Para cada amostra, foram preparados três corpos-de-prova em cada temperatura de sinterização.

A densidade do corpo conformado foi determinada pelo método geométrico (a partir das medidas de dimensão e massa), enquanto a densidade do corpo sinterizado foi determinada pelo método de imersão em líquido (método de Arquimedes). A equação $\mathrm{H}$ foi usada para calcular a densidade dos corpos sinterizados com poros fechados, enquanto os corpos sinterizados porosos, com baixa densidade, foram recobertos (impregnados) com um esmalte cosmético (utilizado para pintar unhas) e, então, a densidade foi determinada com a equação I:

$$
\begin{aligned}
& \rho_{\mathrm{e}}=\frac{\mathrm{m}_{\mathrm{ar}}}{\mathrm{m}_{\mathrm{ar}}-\mathrm{m}_{\text {Liq }}} \rho_{\text {Liq }} \\
& \rho_{\mathrm{e}}=\frac{\mathrm{m}_{\mathrm{ar}}}{\left.\left|\frac{\mathrm{m}_{\text {ar-imp }}-\mathrm{m}_{\text {Liq-imp }}}{\rho_{\text {Liq }}}\right|-\mid \frac{\mathrm{m}_{\text {ar-imp }}-\mathrm{m}_{\mathrm{ar}}}{\rho_{\text {imp }}}\right)}
\end{aligned}
$$

onde $\rho_{\mathrm{e}}$ é a densidade calculada, $\mathrm{m}_{\mathrm{ar}}$ é a massa da amostra medida ao ar, $m_{\text {Liq }}$ é a massa da amostra medida imersa em líquido, $\rho_{\text {Liq }}$ é a densidade do líquido (álcool isopropílico) em função da temperatura, $m_{\text {ar-imp }}$ é a massa da amostra impregnada medida ao ar, $\mathrm{m}_{\text {Liq-imp }}$ é a massa da amostra impregnada medida imersa em líquido e $\rho_{\mathrm{imp}}$ é a densidade do material usado para impregnar a amostra $\left(\rho_{\text {esmalte }} \approx\right.$ $1,31 \mathrm{~g} / \mathrm{cm}^{3}$ ) [22]. Os resultados de densidade foram apresentados em porcentagem de densidade teórica (\% DT), que foi calculada pela regra das misturas usando as densidades de $3,26 \mathrm{~g} / \mathrm{cm}^{3}$ para o AlN e $5,01 \mathrm{~g} / \mathrm{cm}^{3}$ para $\mathrm{O}_{2} \mathrm{O}_{3}$. A perda de massa foi determinada pela diferença entre as massas dos corpos sinterizado e compactado, subtraindo os $2 \%$ em massa de ligante adicionado ao corpo compactado. A análise por difração de raios X (DRX) foi realizada com radiação do cobre $\left(\mathrm{Cu}-\mathrm{k}_{\alpha 1}\right)$ e velocidade de varredura $0,5 \% \mathrm{~min}$ em um difratômetro Rigaku RINT 2000. As análises por DRX foram realizadas nas seções transversais polidas das amostras com alta densidade. Já as amostras com baixa densidade foram moídas em um moinho de anéis de carbeto de tungstênio (Spex, Shatterbox 8500) e, então, compactadas e analisadas por DRX. Os arquivos JCPDS utilizados para identificar as fases cristalinas foram: 25-1133 (AlN), 33-0040 (YAG, 3Y $\mathrm{O}_{3} .5 \mathrm{Al}_{2} \mathrm{O}_{3}$ ), 34-0368 (YAM, 2 $\mathrm{Y}_{2} \mathrm{O}_{3} \cdot \mathrm{Al}_{2} \mathrm{O}_{3}$ ), 33-0041 (YAP, $\mathrm{Y}_{2} \mathrm{O}_{3} \cdot \mathrm{Al}_{2} \mathrm{O}_{3}$ ), 41-1105 $\left(\mathrm{Y}_{2} \mathrm{O}_{3}\right)$ e $76-2495(\mathrm{YN})$. Tanto superfícies de fratura como superfícies polidas dos corpos sinterizados foram analisadas em um microscópio eletrônico de varredura (MEV, Jeol, JSM 6300), sendo as microanálises químicas realizadas com um espectrômetro de energia dispersiva (EDS, Noran) acoplado ao MEV. As amostras foram recobertas com ouro em uma campânula a vácuo e, então, foram analisadas com tensão de trabalho de $15 \mathrm{kV}$.

\section{RESULTADOS}

\section{Densidade}

A densidade relativa dos corpos compactados foi de $55 \%$ DT. Os resultados de densidade relativa em função da temperatura de sinterização são apresentados na Fig. 1. O AlN sem aditivo teve pouca densificação com o aumento da temperatura de sinterização, pois atingiu somente $74 \%$ DT após sinterização a $1850{ }^{\circ} \mathrm{C}$. A adição de $4 \%$ de $\mathrm{Y}_{2} \mathrm{O}_{3}$ (amostra 4Y) deslocou a curva de densificação do AlN para menores temperaturas. A densidade da amostra $4 \mathrm{Y}$ aumentou significativamente com o aumento da temperatura de sinterização, atingindo quase completa densificação após 
sinterização a $1750{ }^{\circ} \mathrm{C}(\sim 97 \% \mathrm{DT}$, Fig. 1). Esta amostra sinterizada a $1800{ }^{\circ} \mathrm{C}$ e $1850{ }^{\circ} \mathrm{C}$ alcançou densidade de 99\%DT.

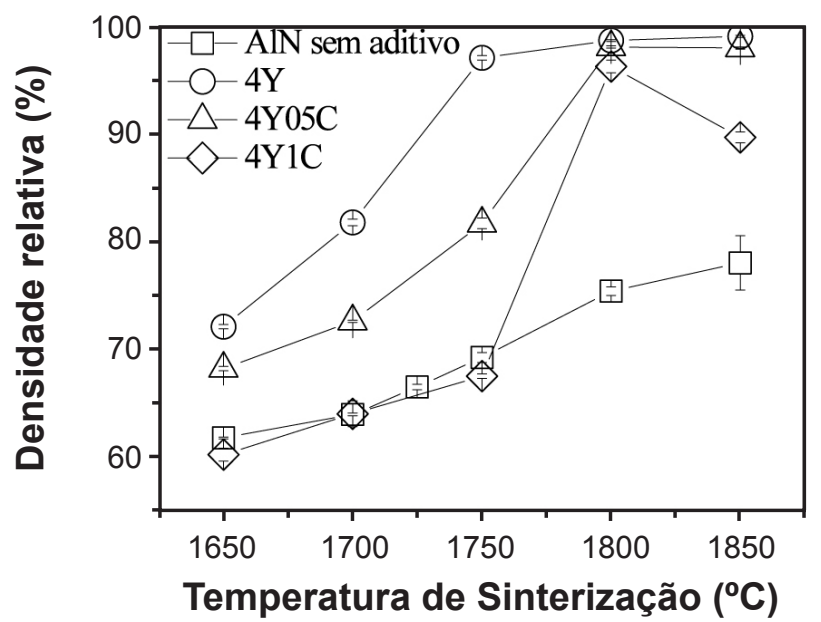

Figura 1: Densidade relativa das amostras 4Y, 4Y05C e 4Y1C em função da temperatura de sinterização.

[Figure 1: Relative density of the $4 Y, 4 Y 05 C$, and $4 Y 1 C$ samples as a function of the sintering temperature.]

Em relação à amostra 4Y, a adição de carbono limitou significativamente a densificação até $1750{ }^{\circ} \mathrm{C}$ (Fig. 1), sendo que as curvas de densificação foram deslocadas para maiores temperaturas de sinterização. A adição de $1 \%$ de carbono praticamente inibiu a ação efetiva do $\mathrm{Y}_{2} \mathrm{O}_{3} \mathrm{em}$ promover a densificação do AlN entre $1650{ }^{\circ} \mathrm{C}$ e $1750^{\circ} \mathrm{C}$, pois as amostras $4 \mathrm{Y} 1 \mathrm{C}$ e AlN sem aditivo tiveram curvas de densificação próximas nesta faixa de temperatura de sinterização (Fig. 1). Porém, a amostra 4Y1C e, também, a amostra 4Y05C atingiram quase completa densificação após sinterização a $1800{ }^{\circ} \mathrm{C}(\sim 99 \% \mathrm{DT})$. Isto mostrou que o aumento de temperatura compensou o efeito prejudicial do carbono na densificação do $\mathrm{AlN} \operatorname{com} \mathrm{Y}_{2} \mathrm{O}_{3}$. O aumento da temperatura de sinterização de $1800{ }^{\circ} \mathrm{C}$ para $1850{ }^{\circ} \mathrm{C}$ não mudou o comportamento de densificação da amostra 4Y05C ( $99 \%$ DT), mas causou uma diminuição significativa de densidade na amostra 4Y1C ( 98\% para $\sim 90 \%$ DT, Fig. 1).

\section{Perda de massa}

Os resultados de perda de massa em função da temperatura de sinterização são apresentados na Fig. 2. A amostra 4Y apresentou perda de massa menor que $1 \%$ entre $1650{ }^{\circ} \mathrm{C}$ e $1850{ }^{\circ} \mathrm{C}$, o que indicou a ocorrência de pouca evaporação de compostos durante sinterização. Já as amostras 4Y05C e 4Y1C apresentaram perdas de massa significativamente maiores do que a amostra $4 \mathrm{Y}$ nesta faixa de temperatura. Isto mostrou que a adição de carbono causou uma evaporação significativa de compostos durante a sinterização do AlN com $\mathrm{Y}_{2} \mathrm{O}_{3}$. A amostra $4 \mathrm{Y} 05 \mathrm{C}$ sinterizada entre $1650{ }^{\circ} \mathrm{C}$ e $1850{ }^{\circ} \mathrm{C}$ apresentou valores de perda de massa na faixa de $2,8 \%$ a $3,7 \%$ (Fig. 2). Estes valores de perda de massa foram maiores do que o valor de perda teórica de $\mathrm{CO}_{2}$ proveniente da reação de oxidação de $0,5 \%$ de carbono $(\sim 2 \%)$. Já a amostra 4Y1C apresentou valores de perda de massa na faixa de $3,3 \%$ a $4,0 \%$ (Fig. 2), cujos valores foram abaixo ou próximos do valor de perda teórica de $\mathrm{CO}_{2}$ proveniente da queima de $1 \%$ de carbono $(\sim 4 \%)$. Estes resultados indicaram que, em relação à amostra $4 \mathrm{Y}$, a adição de carbono causou uma diminuição significativa na quantidade de óxidos formadores de fase líquida durante a sinterização. Após sinterização a $1800{ }^{\circ} \mathrm{C}$, as amostras com carbono apresentaram valores próximos de perda de massa (Fig. 2), o que não aconteceu nas demais temperaturas de sinterização. Isto pode ter influenciado o comportamento de densificação das amostras com carbono, pois as duas amostras atingiram valores próximos de densidade somente na temperatura de $1800{ }^{\circ} \mathrm{C}$ (Fig. 1).

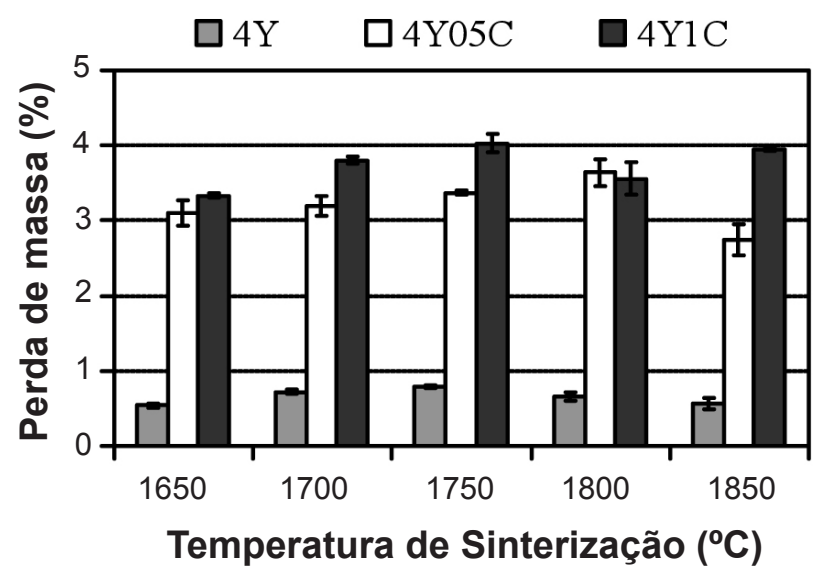

Figura 2: Perda de massa das amostras 4Y, 4Y05C e 4Y1C em função da temperatura de sinterização.

[Figure 2: Weight loss of the 4Y, 4Y05C, and 4Y1C samples as a function of the sintering temperature.]

\section{Evolução das segundas-fases}

Os resultados de DRX das amostras 4Y, 4Y05C e 4Y1C sinterizadas entre $1650{ }^{\circ} \mathrm{C}$ e $1850{ }^{\circ} \mathrm{C}$ são apresentados na Tabela I. A amostra $4 \mathrm{Y}$ sinterizada a $1650{ }^{\circ} \mathrm{C}$ e $1700{ }^{\circ} \mathrm{C}$ apresentou em ordem de preponderância as fases YAG, YAM e YAP. O aumento da temperatura de sinterização causou uma inversão na ordem de preponderância das segundas-fases, pois a amostra $4 \mathrm{Y}$ sinterizada a $1750{ }^{\circ} \mathrm{C}$ apresentou maior quantidade da fase YAP, seguida por YAG e YAM. Após sinterização a $1800{ }^{\circ} \mathrm{C}$ e $1850{ }^{\circ} \mathrm{C}$, a amostra 4Y apresentou em ordem de preponderância somente as fases YAP e YAG (Tabela I). Embora a fase YAP seja estável somente entre $1835{ }^{\circ} \mathrm{C}$ e $1865{ }^{\circ} \mathrm{C}$, [1] este aluminato é freqüentemente identificado em amostras de $\mathrm{AlN}$ com $\mathrm{Y}_{2} \mathrm{O}_{3}$ $[20,23,24]$.

A adição de carbono levou à formação de segundas-fases mais ricas em $\mathrm{Y}_{2} \mathrm{O}_{3}$ em relação à amostra $4 \mathrm{Y}$ (Tabela I). A amostra $4 \mathrm{Y} 05 \mathrm{C}$ apresentou em ordem de preponderância as fases $\mathrm{YAM}$ e $\mathrm{Y}_{2} \mathrm{O}_{3}$ entre $1650{ }^{\circ} \mathrm{C}$ e $1750{ }^{\circ} \mathrm{C}$. Nesta faixa de temperatura, a amostra $4 \mathrm{Y} 1 \mathrm{C}$ apresentou em ordem de preponderância as fases $\mathrm{Y}_{2} \mathrm{O}_{3}$ e $\mathrm{YN}$ (nitreto de ítrio), o que 
Tabela I - Composição das segundas-fases identificadas por DRX das amostras 4Y, 4Y05C e 4Y1C sinterizadas entre $1650{ }^{\circ} \mathrm{C}$ e $1850{ }^{\circ} \mathrm{C}$.

[Table I - Composition of second-phases identified by XRD of the $4 Y, 4 Y 05 C$, and $4 Y 1 C$ samples sintered between $1650^{\circ} \mathrm{C}$ and $\left.1850^{\circ} \mathrm{C}.\right]$

\begin{tabular}{cccc}
\hline \multicolumn{4}{c}{ Segundas-fases em ordem de preponderância } \\
$\mathrm{T}\left({ }^{\circ} \mathrm{C}\right)$ & 4 & Amostras \\
& $4 \mathrm{Y}$ & 4Y05C & 4Y1C \\
\hline 1650 & YAG, YAM, YAP & YAM, $\mathrm{Y}_{2} \mathrm{O}_{3}$ & $\mathrm{Y}_{2} \mathrm{O}_{3}, \mathrm{YN}$ \\
1700 & YAG, YAM, YAP & YAM, $\mathrm{Y}_{2} \mathrm{O}_{3}$ & $\mathrm{Y}_{2} \mathrm{O}_{3}, \mathrm{YN}$ \\
1750 & YAP, YAG, YAM & YAM, $\mathrm{Y}_{2} \mathrm{O}_{3}$ & $\mathrm{Y}_{2} \mathrm{O}_{3}, \mathrm{YN}$ \\
1800 & YAP, YAG & YAP & $\mathrm{YAP}_{2} \mathrm{Y}_{2}$ \\
1850 & YAP, YAG & YAP & $\mathrm{Y}_{2} \mathrm{O}_{3}, \mathrm{YN}$ \\
\hline
\end{tabular}

Nota: $\mathrm{YAG}\left(3 \mathrm{Y}_{2} \mathrm{O}_{3} \cdot 5 \mathrm{Al}_{2} \mathrm{O}_{3}\right), \mathrm{YAM}\left(2 \mathrm{Y}_{2} \mathrm{O}_{3} \cdot \mathrm{Al}_{2} \mathrm{O}_{3}\right)$ e $\mathrm{YAP}\left(\mathrm{Y}_{2} \mathrm{O}_{3} \cdot \mathrm{Al}_{2} \mathrm{O}_{3}\right)$.

mostrou a inibição da formação de fases de aluminatos de ítrio entre $1650{ }^{\circ} \mathrm{C}$ e $1750{ }^{\circ} \mathrm{C}$ com a adição de $1 \%$ de carbono. Estes resultados indicaram que aconteceu uma evaporação preferencial de $\mathrm{Al}_{2} \mathrm{O}_{3}$ durante a sinterização até pelo menos $1750{ }^{\circ} \mathrm{C}$. Isto está de acordo como os resultados de perda de massa (Fig. 2), que indicaram uma evaporação significativa
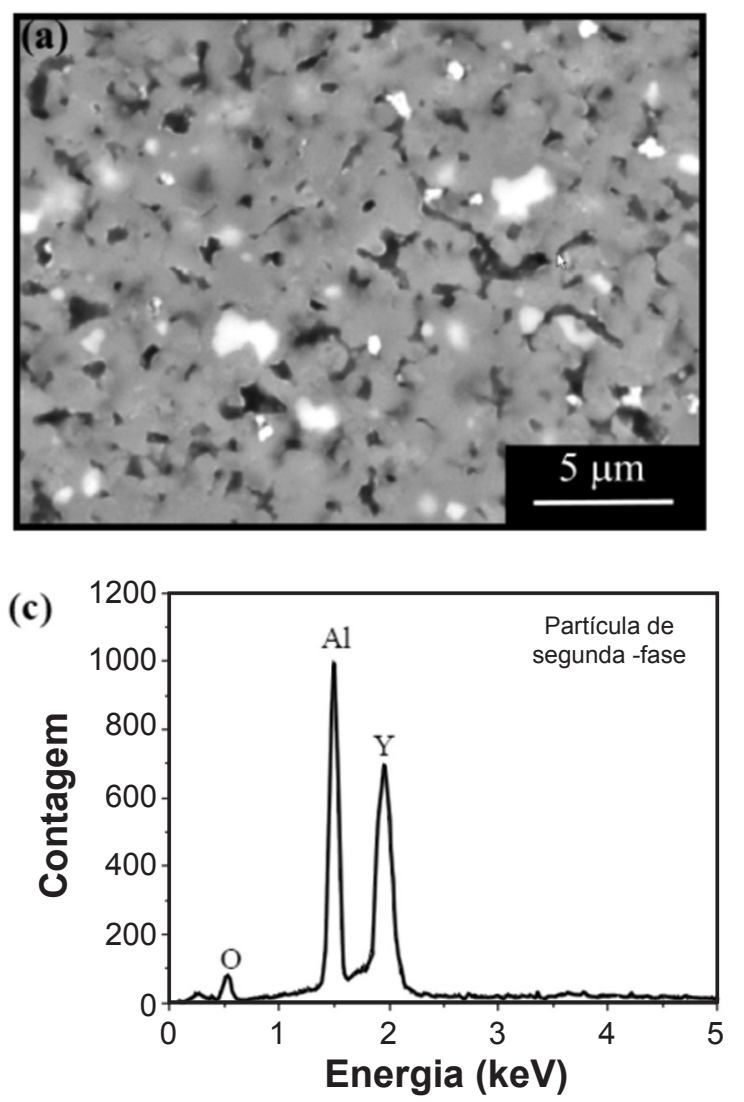

de compostos durante a sinterização das amostras 4Y05C e 4Y1C. O aumento da temperatura de sinterização acima de $1750{ }^{\circ} \mathrm{C}$ levou somente à formação da fase YAP na amostra 4Y05C (Tabela I). Já a amostra 4Y1C sinterizada a $1800{ }^{\circ} \mathrm{C}$ apresentou em ordem de preponderância as fases $\mathrm{YAPe} \mathrm{Y}_{2} \mathrm{O}_{3}$ (Tabela I). Porém, esta amostra voltou a apresentar as fases $\mathrm{Y}_{2} \mathrm{O}_{3}$ e $\mathrm{YN}$ após sinterização a $1850{ }^{\circ} \mathrm{C}$. Estes resultados mostraram que a amostra $4 \mathrm{Y} 1 \mathrm{C}$ apresentou uma mudança significativa na evolução das segundas-fases (Tabela I), pois aconteceu a formação de uma fase de aluminato de ítrio após sinterização a $1800{ }^{\circ} \mathrm{C}$, que não foi observada nas demais temperaturas de sinterização. A $1800{ }^{\circ} \mathrm{C}$, as duas amostras com adição de carbono atingiram valores de densidade próximos daquele observado para a amostra sem carbono (Fig. 1). Os resultados indicaram que a densificação do AlN com $\mathrm{Y}_{2} \mathrm{O}_{3}$ foi favorecida pela formação de fases de aluminatos de ítrio, onde o aumento da fração de $\mathrm{Al}_{2} \mathrm{O}_{3}$ na composição destes aluminatos promoveu a densificação em mais baixas temperaturas de sinterização, como pode ser observado pela formação das fases YAG e YAM na amostra 4Y (Tabela I).

\section{Evolução microestrutural}

A Fig. 3 mostra imagens de MEV e um espectro de EDS
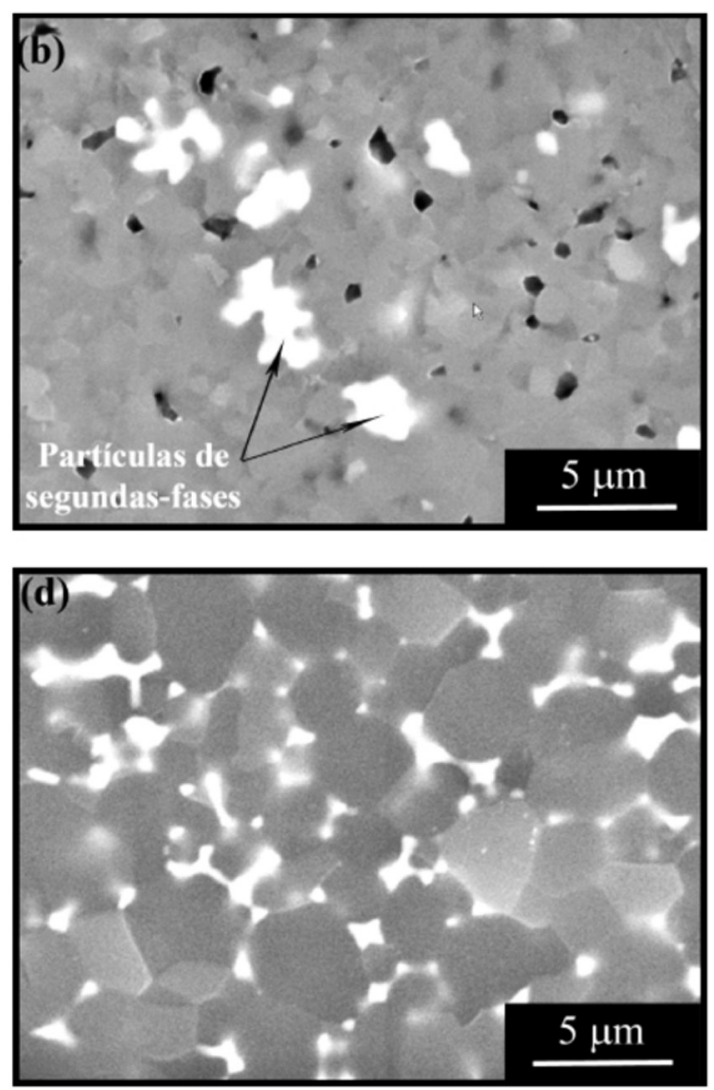

Figura 3: Imagens de MEV (elétrons secundários) das superfícies polidas da amostra $4 \mathrm{Y}$ sinterizada a $1700{ }^{\circ} \mathrm{C}$ (a), $1750{ }^{\circ} \mathrm{C}(\mathrm{b})$ e $1850{ }^{\circ} \mathrm{C}$ (d). Espectro de EDS de uma partícula de segunda-fase observada na amostra 4 Y sinterizada a $1750{ }^{\circ} \mathrm{C}$ (c).

[Figure 3: SEM images (secondary electrons) of polished surfaces of the $4 Y$ sample sintered at $1700{ }^{\circ} \mathrm{C}(\mathrm{a}), 1750{ }^{\circ} \mathrm{C}(\mathrm{b})$, and $1850{ }^{\circ} \mathrm{C}(\mathrm{d})$. EDS spectrum of a second-particle observed in the $4 Y$ sample sintered at $1750{ }^{\circ} \mathrm{C}(\mathrm{c})$.] 

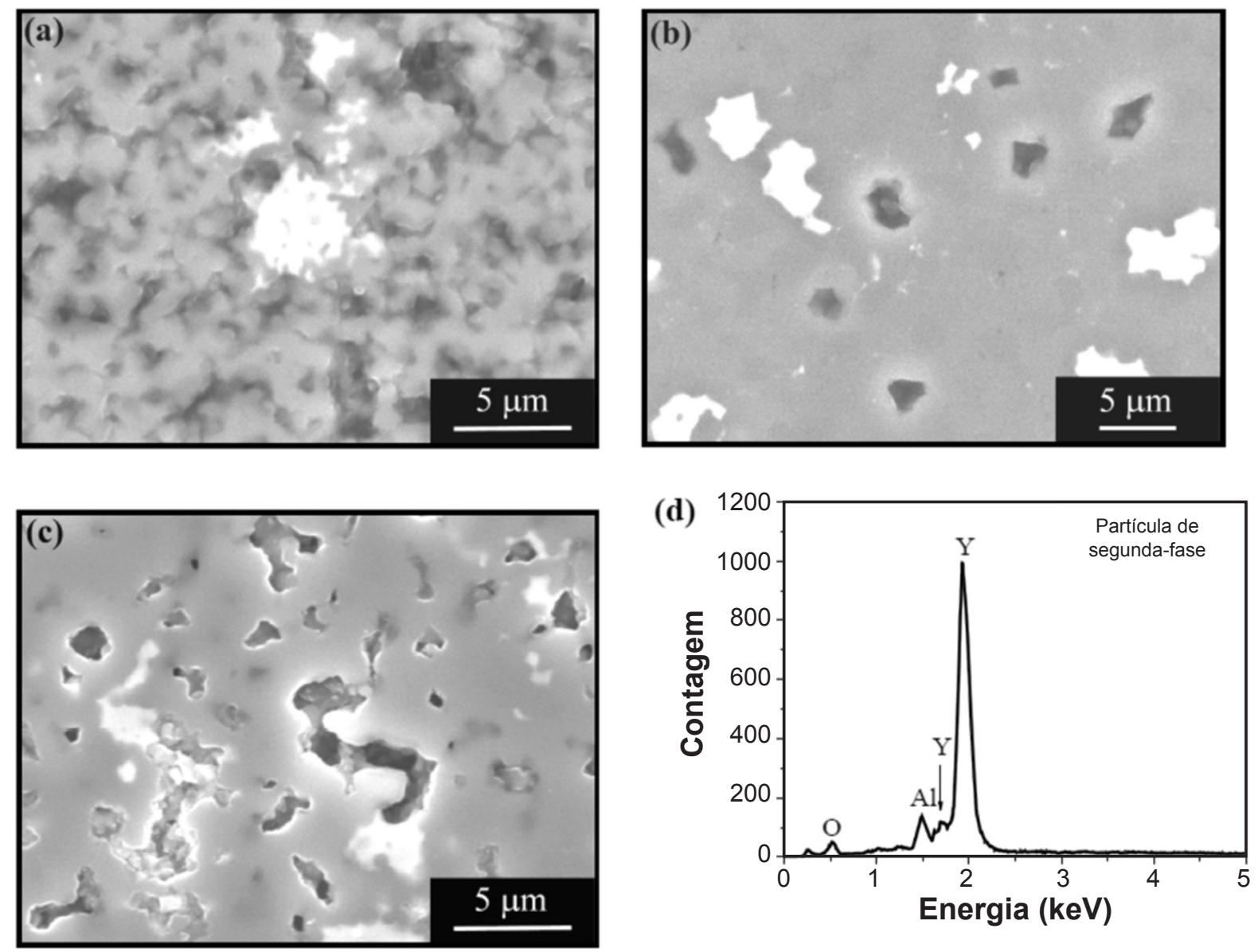

Figura 4: Imagens de MEV (elétrons secundários) das superfícies polidas das amostras com adição de carbono: amostra 4Y05C sinterizada a $1750{ }^{\circ} \mathrm{C}$ (a) e amostra 4 Y $1 \mathrm{C}$ sinterizada a $1800{ }^{\circ} \mathrm{C}$ (b) e $1850{ }^{\circ} \mathrm{C}$ (c). Espectro de EDS de uma partícula de segunda-fase observada na amostra 4Y1C sinterizada a $1750{ }^{\circ} \mathrm{C}(\mathrm{d})$.

[Figure 4: SEM images (secondary electrons) of polished surfaces of the samples with addition of carbon: 4Y05C sample sintered at $1750{ }^{\circ} \mathrm{C}(\mathrm{a})$ and $4 Y 1 \mathrm{C}$ sintered at $1800^{\circ} \mathrm{C}(\mathrm{b})$ and $1850^{\circ} \mathrm{C}(\mathrm{c})$. EDS spectrum of a second-phase particle observed in the $4 Y 1 \mathrm{C}$ sample sintered at $\left.1750{ }^{\circ} \mathrm{C}(d).\right]$

da amostra 4Y. Após sinterização a $1700{ }^{\circ} \mathrm{C}$, esta amostra apresentou uma microestrutura com poros interconectados (Fig. 3a), onde se observou somente o crescimento de pescoços entre as partículas primárias de $\mathrm{AlN}$, que é uma característica microestrutural associada com o estágio intermediário da sinterização no estado sólido. Nesta temperatura de sinterização, a amostra $4 \mathrm{Y}$ apresentou cerca de $80 \%$ DT (Fig. 1). Porém, observou-se uma microestrutura praticamente densa após sinterização a 1750 ${ }^{\circ} \mathrm{C}$ (Fig. 3b). Esta rápida alteração microestrutural indicou a ocorrência de densificação por sinterização via fase líquida. Isto é plausível, pois a temperatura de formação de fase líquida para o AlN com $\mathrm{Y}_{2} \mathrm{O}_{3}$ pode ocorrer a partir de aproximadamente $1720{ }^{\circ} \mathrm{C}$, quando a fase YAG está presente [1,2].

Partículas de segundas-fases foram observadas na amostra $4 \mathrm{Y}$ sinterizada entre $1650{ }^{\circ} \mathrm{C}$ e $1800{ }^{\circ} \mathrm{C}$ (Figs. 3a e 3 b), como relatado em trabalhos anteriores [1,2]. Estas partículas grandes de segundas-fases são decorrentes da utilização de pó de $\mathrm{Y}_{2} \mathrm{O}_{3}$ com distribuição granulométrica mais larga do que a do pó de AlN [1,2]. A análise por EDS destas partículas revelou a presença de $\mathrm{O}, \mathrm{Al}$ e $\mathrm{Y}$ (Fig. 3c), o que mostrou serem partículas de aluminatos de ítrio, conforme resultados de DRX (Tabela I). Em trabalhos anteriores [1,2], identificou-se que estas partículas são compostas por aluminatos de ítrio mais refratários (YAM ou YAP) do que a fase YAG. Estas partículas fundiram e penetraram (molharam) entre os grãos de AlN somente na temperatura de sinterização de $1850{ }^{\circ} \mathrm{C}$ (Fig. 3d). As amostras 4 Y05C e $4 \mathrm{Y} 1 \mathrm{C}$ sinterizadas a $1750{ }^{\circ} \mathrm{C}$ apresentaram microestruturas com poros interconectados e características típicas do estágio intermediário de sinterização no estado sólido (Fig. 4a), similares àquela observada na amostra $4 \mathrm{Y}$ sinterizada a $1700{ }^{\circ} \mathrm{C}$ (Fig. 3a). No entanto, estas amostras apresentaram microestruturas praticamente densas após sinterização a $1800{ }^{\circ} \mathrm{C}$ (Fig. 4b), indicando que os mecanismos de sinterização via fase líquida foram ativados nesta temperatura, o que levou a um processo de rápida densificação. Estes resultados mostraram que a adição de carbono aumentou a temperatura de formação da fase líquida, o que levou ao atraso na densificação do AlN com $\mathrm{Y}_{2} \mathrm{O}_{3}$. Porosidade residual foi observada nas amostras 
4Y05C e 4Y1C sinterizadas a $1800{ }^{\circ} \mathrm{C}$ (Fig. 4b), o que explica a pequena diferença de densidade entre as amostras sem e com carbono sinterizadas a $1800{ }^{\circ} \mathrm{C}$ (Fig. 1). Acima desta temperatura de sinterização, a amostra 4Y05C continuou com uma microestrutura densa, mas a amostra 4Y1C apresentou um aumento significativo de poros (Fig. $4 c)$, que resultou na sua diminuição de densidade entre $1800{ }^{\circ} \mathrm{C}$ e $1850{ }^{\circ} \mathrm{C}$ (Fig. 1).

Partículas grandes de segundas-fases também foram observadas nas amostras 4Y05C e 4Y1C sinterizadas até $1800{ }^{\circ} \mathrm{C}$ (Figs. 4a e 4b). Na amostra 4Y05C, as partículas de segundas-fases exibiram espectros de EDS similares ao observado na amostra 4Y (Fig. 3c), além de mostrar a mesma tendência para penetrar entre os grãos de AlN na temperatura de sinterização de $1850^{\circ} \mathrm{C}$ (Fig. 3d), indicando serem de fases YAM ou YAP, conforme resultados da análise por DRX (Tabela I). Entretanto, a amostra 4Y1C continuou a apresentar partículas grandes de segundas-fases acima de $1800{ }^{\circ} \mathrm{C}$ (Fig. 4c). A análise por EDS das partículas de segundas-fases da amostra $4 \mathrm{Y} 1 \mathrm{C}$ revelou a presença de alto teor de $\mathrm{Y}$ e baixos teores de $\mathrm{O}$ e $\mathrm{Al}$ (Fig. 4d), o que indicou serem de fase $\mathrm{Y}_{2} \mathrm{O}_{3}$, possivelmente de partículas originais não reagidas durante a sinterização, conforme resultados de DRX (Tabela I), exceto quando sinterizada a $1800{ }^{\circ} \mathrm{C}$, pois nesta temperatura o espectro de EDS foi similar àquele obtido para as amostras 4Y e 4Y05C (Fig. 3c). Estes resultados indicaram que a densificação da amostra $4 \mathrm{Y} 1 \mathrm{C}$ foi limitada acima de $1800{ }^{\circ} \mathrm{C}$ (Figs. 1 e $4 \mathrm{c}$ ) devido à presença de fases $\mathrm{Y}_{2} \mathrm{O}_{3}$ e YN (Tabela I) mais refratárias do que os aluminatos de ítrio.

\section{DISCUSSÃO}

\section{Evolução das segundas-fases}

Em trabalho anterior verificou-se que o AlN com $\mathrm{Y}_{2} \mathrm{O}_{3}$, quando sinterizado sob atmosfera não redutora, apresenta indícios significativos de evaporação de segundas-fases somente acima de $1900{ }^{\circ} \mathrm{C}$ [1]. Foi indicado que os compostos $\mathrm{Al}_{2} \mathrm{O}_{3}$ e $\mathrm{Y}_{2} \mathrm{O}_{3}$ podem evaporar durante a sinterização do $\mathrm{AlN}$ sob atmosfera não redutora, mas a taxa de evaporação do $\mathrm{Al}_{2} \mathrm{O}_{3}$ aumenta mais rápido com a temperatura, principalmente acima de $1850^{\circ} \mathrm{C}$ [23]. Deste modo, a evolução de segundas-fases do AlN com $\mathrm{Y}_{2} \mathrm{O}_{3}$ sinterizado sob atmosfera não redutora mostra uma tendência para formar fases mais ricas em $\mathrm{Y}_{2} \mathrm{O}_{3}$ com o aumento da temperatura de sinterização, como observado na amostra 4Y (Tabela I). Os resultados do presente trabalho (amostra 4Y, Tabela I), bem como os reportados [1, 23], mostraram que as amostras sinterizadas em altas temperaturas tendem a apresentar segundas-fases com composição igual ou próxima da fase YAP. Como esta fase é mais estável do que os demais aluminatos (YAG e YAM) acima de $1835^{\circ} \mathrm{C}$ [25], pode-se dizer que, durante a sinterização do AlN em altas temperaturas, a fase líquida tende a perder mais $\mathrm{Al}_{2} \mathrm{O}_{3}$ para atingir uma composição próxima da fase YAP. A evaporação preferencial de $\mathrm{Al}_{2} \mathrm{O}_{3}$ pode acontecer durante a sinterização do AlN com $\mathrm{Y}_{2} \mathrm{O}_{3}$, pois a pressão parcial de oxigênio do $\mathrm{Al}_{2} \mathrm{O}_{3}$ é maior do que aquela observada para o $\mathrm{Y}_{2} \mathrm{O}_{3}[12,20]$. Estes resultados indicaram que, independente do tipo de atmosfera de sinterização (redutora ou não), pode ocorrer evaporação de compostos, principalmente durante a sinterização em altas temperaturas $\left(>1800{ }^{\circ} \mathrm{C}\right)$. Porém, a evaporação de compostos foi considerada baixa, já que os valores de perda de massa da amostra 4Y sinterizada entre $1650{ }^{\circ} \mathrm{C}$ e $1850{ }^{\circ} \mathrm{C}$ foram menores do que $1 \%$ (Fig. 2).

Durante a sinterização do AlN com $\mathrm{Y}_{2} \mathrm{O}_{3}$ sob atmosfera redutora, o processo de desoxidação das segundasfases tem sido proposto para ocorrer pela desoxidação e nitretação inicial dos aluminatos de ítrio, seguida pela desoxidação e nitretação das fases remanescentes $\left(\mathrm{Al}_{2} \mathrm{O}_{3}\right.$ e $\mathrm{Y}_{2} \mathrm{O}_{3}$ ), conforme sugerido pelas reações A a C. Quando pó de grafita ou de negro de fumo é usado como aditivo de sinterização, o processo de desoxidação e nitretação do AlN com $\mathrm{Y}_{2} \mathrm{O}_{3}$ pode proceder de acordo com a reação $E[19,20]$ ou de acordo com as reações $E$ a $G$ [12]. No presente trabalho, propõe-se um mecanismo alternativo para o processo de desoxidação por carbono e nitretação dos compostos durante a sinterização do $\mathrm{AlN}$ com $\mathrm{Y}_{2} \mathrm{O}_{3}$. Com base nos resultados de DRX (Tabela I), observa-se que o mecanismo de desoxidação e nitretação do AlN com adição simultânea de $\mathrm{Y}_{2} \mathrm{O}_{3}$ e $\mathrm{C}$ pode proceder de acordo com as reações E e G. Para a amostra 4Y05C sinterizada entre $1650^{\circ} \mathrm{C}$ e $1750{ }^{\circ} \mathrm{C}$, observou-se a presença das fases YAM e $\mathrm{Y}_{2} \mathrm{O}_{3}$ (Tabela I), o que sugere que a adição de $0,5 \%$ de $\mathrm{C}$ causou a desoxidação de parte do $\mathrm{Al}_{2} \mathrm{O}_{3}$ contido no pó de AlN (reação E), resultando na formação de um aluminato rico em $\mathrm{Y}_{2} \mathrm{O}_{3}$ (YAM) e na presença de $\mathrm{Y}_{2} \mathrm{O}_{3}$ não reagido. Porém, quando se adicionou $1 \%$ de carbono, observaram-se as fases $\mathrm{Y}_{2} \mathrm{O}_{3}$ e YN após a sinterização entre $1650{ }^{\circ} \mathrm{C}$ e $1750{ }^{\circ} \mathrm{C}$ (amostra 4Y1C, Tabela I), o que indicou que a adição em excesso de carbono causou a completa desoxidação e nitretação do $\mathrm{Al}_{2} \mathrm{O}_{3}$ contido no pó de $\mathrm{AlN}$ (reação E), bem como a parcial desoxidação e nitretação do $\mathrm{Y}_{2} \mathrm{O}_{3}$ adicionado (reação $\mathrm{G}$ ). Isto mostra que o processo de desoxidação da superfície do pó de AlN ocorre antes da reação entre $\mathrm{Al}_{2} \mathrm{O}_{3}$ e $\mathrm{Y}_{2} \mathrm{O}_{3}$ para formação dos aluminatos de ítrio, diferente de mecanismo proposto [12]. Portanto, a reação $\mathrm{F}$ não é prevista para acontecer durante a sinterização do AlN com adição simultânea de $\mathrm{Y}_{2} \mathrm{O}_{3}$ e C.

\section{Densificação}

Em relação ao AlN sem aditivo, a adição de $4 \%$ de $\mathrm{Y}_{2} \mathrm{O}_{3}$ (amostra 4Y) deslocou a curva de densificação para menores temperaturas (Fig. 1), o que mostrou que este aditivo aumentou significativamente a sinterabilidade desta cerâmica. Esta melhora no comportamento de densificação do AlN com $\mathrm{Y}_{2} \mathrm{O}_{3}$ pode ser atribuída à formação de fase líquida em torno de $1720{ }^{\circ} \mathrm{C}[1,2]$, pois esta temperatura corresponde à temperatura eutética $\left(\mathrm{L} \rightarrow \mathrm{YAG}+\alpha-\mathrm{Al}_{2} \mathrm{O}_{3}\right)$ mais baixa prevista no diagrama de fase do sistema ternário AlN- $\mathrm{Y}_{2} \mathrm{O}_{3}-\mathrm{Al}_{2} \mathrm{O}_{3}$ [9]. Assim, a amostra $4 \mathrm{Y}$ atingiu densidade de $97 \%$ DT a partir de $1750{ }^{\circ} \mathrm{C}$ (Fig. 1) porque sua densificação ocorreu por sinterização via fase líquida 
acima de $1720^{\circ} \mathrm{C}$. Por outro lado, a amostra 4Y1C apresentou um comportamento de densificação próximo daquele observado para o AlN sem aditivo abaixo de $1800{ }^{\circ} \mathrm{C}$ (Fig. 1). Estes resultados indicaram que a adição de $1 \%$ de carbono praticamente inibiu a ação efetiva do $\mathrm{Y}_{2} \mathrm{O}_{3}$ em promover densificação no AlN em baixas temperaturas de sinterização. Como a amostra 4Y1C não apresentou fases de aluminato de ítrio entre $1650{ }^{\circ} \mathrm{C}$ e $1750{ }^{\circ} \mathrm{C}$ (Tabela I) e sabendo-se que a formação de aluminatos de ítrio promove uma densificação significativa no AlN por sinterização no estado sólido [1, 2, 26], pode-se dizer que o AlN apresentou pouca densificação em decorrência da não formação de aluminatos de ítrio durante a sinterização até $1750^{\circ} \mathrm{C}$. Estes resultados confirmaram que a formação de aluminatos de ítrio e a subseqüente reação destas segundas-fases com o AlN podem induzir uma significativa densificação no AlN antes da formação de fase líquida ( 80\% DT, Fig. 1), como relatado em trabalhos anteriores [1,2]. Estes resultados reforçam a interpretação de que a adição de carbono causa a desoxidação do $\mathrm{Al}_{2} \mathrm{O}_{3}$ presente na superfície do pó de AlN, o que pode inibir a formação de aluminatos, caso não haja $\mathrm{Al}_{2} \mathrm{O}_{3}$ suficiente para reagir com o aditivo de sinterização $\left(\mathrm{Y}_{2} \mathrm{O}_{3}\right)$.

As amostras $4 \mathrm{Y} 05 \mathrm{Ce} 4 \mathrm{Y} 1 \mathrm{C}$ não apresentaram a fase YAG entre $1650{ }^{\circ} \mathrm{C}$ e $1750{ }^{\circ} \mathrm{C}$ (Tabela I) e, portanto, não ocorreu formação de fase líquida a partir de $1720{ }^{\circ} \mathrm{C}$, limitando a densificação em relação à amostra 4Y (Fig. 1). As amostras com carbono sinterizadas até $1750{ }^{\circ} \mathrm{C}$ apresentaram microestruturas com poros interconectados (Fig. 4a), confirmando que não houve formação de fase líquida até esta temperatura, pois a presença de poros interconectados é uma característica microestrutural do estágio intermediário da sinterização no estado sólido. Corrobora esta assertiva, o fato de que o aumento do teor de $\mathrm{Y}_{2} \mathrm{O}_{3}$, acima de $5 \%$ em massa, causou um atraso significativo na densificação do AlN [27, 28]. Sabendo-se que a composição das segundasfases varia em função dos teores de aditivo de sinterização e de oxigênio presente na superfície do pó de AIN [23], a adição em excesso de $\mathrm{Y}_{2} \mathrm{O}_{3}$ pode levar à formação de fases mais ricas em $\mathrm{Y}_{2} \mathrm{O}_{3}$, ou seja, mais refratárias do que a fase YAG. Isto aumenta a temperatura de formação da fase líquida durante a sinterização, resultando no atraso da densificação, isto é, no aumento da temperatura de sinterização. Estes resultados reforçam a interpretação de que a formação da fase YAG pode levar à formação de fase líquida ao redor de $1720^{\circ} \mathrm{C}$ e, então, promover a completa densificação do AlN em temperaturas tão baixas quanto $1800{ }^{\circ} \mathrm{C}$ (Fig. 1). As amostras 4Y e 4Y05C apresentaram microestruturas com partículas de segundas-fases após sinterização entre $1650{ }^{\circ} \mathrm{C}$ e $1800{ }^{\circ} \mathrm{C}$ (Figs. 3a, 3b, 4a e 4b). Outros trabalhos [10, 16, 24] também evidenciaram partículas grandes de segundas-fases no AlN preparado com $\mathrm{Y}_{2} \mathrm{O}_{3}$ ou óxidos de terras raras sinterizado abaixo de $1850{ }^{\circ} \mathrm{C}$. Estas partículas de segundas-fases apresentaram composição mais refratária (YAM ou YAP) do que a fase YAG e fundiram somente acima de $1800{ }^{\circ} \mathrm{C}$ (Fig. 3d) [1, 2], pois as fases YAM e YAP são associadas com fase líquida acima desta temperatura de sinterização, como previsto no diagrama de fase do sistema $\mathrm{Al}_{2} \mathrm{O}_{3}-\mathrm{Y}_{2} \mathrm{O}_{3}$ [25]. Na amostra 4 Y1C sinterizada a $1850{ }^{\circ} \mathrm{C}$ (Fig. 4c), as partículas de segundas-fases foram associadas com a fase $\mathrm{Y}_{2} \mathrm{O}_{3}$ (Tabela I e Fig. 4d) e, portanto, permaneceram na microestrutura até esta temperatura de sinterização, pois esta fase $\left(\mathrm{Y}_{2} \mathrm{O}_{3}\right)$ tem maior temperatura de fusão do que as fases de aluminatos de ítrio, conforme previsto no diagrama de fase do sistema $\mathrm{Al}_{2} \mathrm{O}_{3}-\mathrm{Y}_{2} \mathrm{O}_{3}$ [25]. Como as amostras $4 \mathrm{Y} 05 \mathrm{C}$ e $4 \mathrm{Y} 1 \mathrm{C}$ atingiram densidades elevadas, próximas daquela observada para a amostra $4 \mathrm{Y}$, após a sinterização na temperatura de $1800{ }^{\circ} \mathrm{C}$ (Fig. 1), pode-se dizer que pequenas frações de fase líquida foram suficientes para promover a densificação do AlN com $\mathrm{Y}_{2} \mathrm{O}_{3}$. Embora tenha ocorrido elevada evaporação de compostos com a adição de carbono (Fig. 2), as amostras 4Y05C e 4Y1C não apresentaram evidências da ocorrência de aprisionamento de gás no interior dos poros fechados, que pode dificultar a densificação em decorrência da estabilização dos poros durante a sinterização em altas temperaturas [29]. Estes resultados indicaram que a densificação do AlN não foi praticamente influenciada pela quantidade de fase líquida presente durante a sinterização e pelo aprisionamento de gás nos poros fechados. Portanto, a densificação do AlN com $\mathrm{Y}_{2} \mathrm{O}_{3}$ foi influenciada, principalmente, pela temperatura de formação de fase líquida, pois as amostras com carbono apresentaram pouca densificação abaixo de $1800{ }^{\circ} \mathrm{C}$ em decorrência da formação de segundas-fases mais refratárias em relação à amostra sem carbono (Tabela I).

\section{CONCLUSÕES}

Os resultados deste estudo, no qual se verificou o mecanismo de evolução de segundas-fases e o efeito da composição das segundas-fases na densificação do AlN com $4 \%$ de $\mathrm{Y}_{2} \mathrm{O}_{3}$ entre $1650{ }^{\circ} \mathrm{C}$ e $1850{ }^{\circ} \mathrm{C}$, onde a variação da composição foi induzida pela adição de $0,5 \%$ e $1 \%$ em massa de carbono, levaram às seguintes conclusões: a adição de carbono induz à formação de fases refratárias ricas em ítrio, que retarda a densificação do AlN devido ao aumento da temperatura de formação de fase líquida. A formação de segundas-fases ricas em ítrio é decorrente da desoxidação e nitretação do $\mathrm{Al}_{2} \mathrm{O}_{3}$ presente na superfície do pó de $\mathrm{AlN}$, o que inibe a formação de aluminatos ricos em $\mathrm{Al}_{2} \mathrm{O}_{3}$, os quais apresentam menores temperaturas de fusão; as espécies voláteis geradas pela desoxidação com carbono não permaneceram aprisionadas no interior dos poros fechados durante a sinterização em altas temperaturas e, portanto, não influenciaram o comportamento de densificação do AlN com $\mathrm{Y}_{2} \mathrm{O}_{3}$; a diminuição da fração de segundas-fases líquidas, causada pela desoxidação por carbono, não influencia a densificação do AlN com $\mathrm{Y}_{2} \mathrm{O}_{3}$ no processo de sinterização assistida por fase líquida.

\section{AGRADECIMENTOS}

Os autores agradecem à FAPESP e ao CNPq pelo suporte financeiro. 


\section{REFERÊNCIAS}

[1] A. L. Molisani, H. N. Yoshimura, H. Goldenstein, Cerâmica 52, 322 (2006) 151.

[2] A. L. Molisani, H. N. Yoshimura, H. Goldenstein, J. Mater. Sci.: Mater. Electron. 20 (2009) 1.

[3] H. N. Yoshimura, A. L. Molisani, N. E. Narita, P. C. Cesar, H. Goldenstein, Mater. Res. 10, 2 (2007) 127.

[4] H. N. Yoshimura, N. E. Narita, A. L. Molisani, H. Goldenstein, J. Mater. Sci. 44, 21 (2009) 5773.

[5] J. Li, M. Nakamura, T. Shirai, K. Matsumaru, C. Ishizaki, K. Ishizaki, J. Am. Ceram. Soc. 89 (2006) 937.

[6] A. Kocjan, K. Krnel, T. Kosma, J. Eur. Ceram. Soc. 28 (2008) 1003.

[7] G. A. Slack, R. A. Tanzilli, R. O. Pohl, J. W. Vandersande, J. Phys. Chem. Solids 48, 7 (1987) 641.

[8] A. Al-Shaikhi, G. P. Srivastava, J. Appl. Phys. 103 (2008) 083554.

[9] K. Watari, H. J. Hwang, M. Toriyama, S. Kanzaki, J. Mater. Res. 14, 4 (1999) 1409.

[10] A. V. Virkar, T. B. Jackson, K. L. More, R. A. Cutler, J. Am. Ceram. Soc. 80 (1997) 1421.

[11] K. Watari, M. Kawamoto, K. Ishizaki, J. Mater. Sci. 26 (1991) 4727.

[12] T. Nakamatsu, F. Pomar, K. Ishizaki, J. Mater. Sci. 34 (1999) 1553.

[13] K. Watari, H. Nakano, K. Urabe, K. Ishizaki, S. X. Cao, K. Mori, J. Mater. Res. 17, 11 (2002) 2940.

[14] H. Nakano, K. Watari, H. Hayashi, K. Urabe, J. Am. Ceram. Soc. 85, 12 (2002) 3093.
[15] H. Nakano, K. Watari, K. Urabe, J. Am. Ceram. Soc. 23, 10 (2003) 1761.

[16] M. Kasori, F. Ueno, J. Eur. Ceram. Soc. 15, 5 (1995) 435.

[17] A. M. Hundere, M. A. Einarsrud, J. Eur. Ceram. Soc. 16, 8 (1996) 899.

[18] R.-R. Lee, J. Am. Ceram. Soc. 74, 9 (1991) 2242.

[19] G.-C. Lai, Y. Nagai, J. Ceram. Soc. Japan 103, 1 (1995) 6. [20] H. Yan, W. R. Cannon, D. J. Shanefield, J. Am. Ceram. Soc. 76, 1 (1993) 166.

[21] K.-H. Lin, Y.-C. Lin, S.-T. Lin, J. Mater. Process. Technol. 201 (2008) 701.

[22] A. L. Molisani, Sinterização e caracterização de propriedades mecânicas de cerâmicas de nitreto de alumínio, Tese Dr., Escola Politécnica da Universidade de S. Paulo, S. Paulo, SP (2009) 63.

[23] S. Mitra, G. Dutta, I. Dutta, J. Am. Ceram. Soc. 78, 9 (1995) 2335.

[24] P. S. De Baranda, A. K. Knudsen, E. Ruh, J. Am. Ceram. Soc. 77, 7 (1994) 1846.

[25] E. M Levin, C. R. Robbins, H. F. McMurdie, Phase diagrams for ceramists, The Am. Ceram. Soc., EUA (1964). [26] M. Tajika, W. Rafaniello, K. Niihara, Mater. Lett. 46 (2000) 98.

[27] N. H. Kim, Q. D. Fun, K. Komeya, T. Meguro, J. Am. Ceram. Soc. 79, 10 (1996) 2645.

[28] J. Jarrige, K. Bouzouita, C. Doradoux, M. Billy, J. Eur. Ceram. Soc. 12, 4 (1993) 279.

[29] W. Dong, H. Jain, M. P. Harmer, J. Am. Ceram. Soc. 88, 7 (2005) 1714.

(Rec. 25/01/2010, Ac. 20/02/2010) 